\title{
ON DIFFERENCE METHODS FOR THE SOLUTION OF A CAUCHY PROBLEM FOR A HYPERBOLIC EQUATION WITH DATA ON A PARABOLIC LINE ( ${ }^{1}$ )
}

\author{
BY \\ HAJIMU OGAWA
}

1. Introduction. In this paper we consider the problem of solving the Cauchy problem for the partial differential equation

$$
L u=K(y) \frac{\partial^{2} u}{\partial x^{2}}+\frac{\partial^{2} u}{\partial y^{2}}+a(x, y) \frac{\partial u}{\partial x}+b(x, y) \frac{\partial u}{\partial y}+c(x, y) u=f(x, y)
$$

by finite difference methods. We assume that $K$ is a monotone increasing function of $y$ with $K(0)=0$. For $y<0$, the equation (1.1) is hyperbolic, with characteristics given by the two families

$$
\begin{aligned}
& d y / d x=(-K)^{-1 / 2} \\
& d y / d x=-(-K)^{-1 / 2}
\end{aligned}
$$

Let $A$ and $B$ be two points on the $x$-axis with $x_{A}<x_{B}$. We denote by $D$ the open domain bounded by the segment $A B$ of the $x$-axis, the characteristic $\Gamma_{1}$ of the family (1.2b) passing through $A$, and by the characteristic $\Gamma_{2}$ of the family (1.2a) passing through $B$. We assume that $K$ is of class $C^{3}(\bar{D})$, $a$ and $b$ are of class $C^{1}(\bar{D})$ and $c$ is of class $C(\bar{D})$. The Cauchy problem which we investigate is that of finding a solution of (1.1) in $D$ which satisfies the initial conditions

$$
u(x, 0)=\psi_{1}(x), \quad \frac{\partial u}{\partial y}(x, 0)=\psi_{2}(x)
$$

on the parabolic segment $A B$.

An approximation $U$ to the solution $u$ of (1.1) is found as the solution to an initial value problem for a difference equation on a mesh region depending on the original domain $D$. If $u$ exists and belongs to class $C^{2}(\bar{D})$ and if the solution of the difference equation satisfies a maximum principle for all sufficiently small mesh widths, it is shown that $U$ tends uniformly to $u$ as the mesh size tends to zero. As in the author's [1] investigation of the Tricomi problem, it is found that the conditions for a differential-equation maximum principle, as found by Weinberger [2] and Protter [3], imply the conditions

Received by the editors February 8, 1961.

(1) This paper is part of a doctoral dissertation presented at the University of California, Berkeley. The author is indebted to his thesis director, Professor M. H. Protter, for his valuable advice and encouragement. 
for a difference-equation maximum principle, except near the $x$-axis where additional conditions are required.

2. The difference problem. We write the characteristics of (1.1) in the form

$$
x-x_{0}= \pm G(y)
$$

where

$$
G(y)=\int_{y}^{0}[-K(\eta)]^{1 / 2} d \eta ; \quad y \leqq 0 .
$$

Let $-H(x)$ be the inverse function to $G(y)$. Then the relation between $H$ and $K$ is given by

$$
H^{\prime}(x)=[-K(y)]^{-1 / 2}
$$

at each point $(x, y)$ of the curve $y=-H(x)$. Thus $H$ has four continuous derivatives and $H(x)>0, H^{\prime}(x)>0$ and $H^{\prime \prime}(x)<0$ for $0<x \leqq\left(x_{B}-x_{A}\right) / 2$.

We divide the segment $A B$ into $N$ equal parts, each of length $h$ and through each of the points $x_{k}=x_{A}+k h(k=1,2, \cdots, N-1)$ on $A B$ we draw both characteristics. These characteristics, together with the characteristics $\Gamma_{1}$ and $\Gamma_{2}$, intersect at the points

$$
\left(x_{k}+\frac{n h}{2},-y_{n}\right) ; \quad k=0,1, \cdots, N-n ; n=1,2, \cdots, N,
$$

with the ordinates satisfying

$$
G\left(-y_{n}\right)=\frac{n h}{2} ; \quad n=1,2, \cdots, N .
$$

Let us denote by $\bar{D}_{h}$ the set of points given by (2.4) together with the points $x_{k}(k=1,2, \cdots, N-1)$ on $A B$. If we take $y_{0}=0$, and associate with each point $\left(x_{k}+n h / 2,-y_{n}\right)$ the pair $(k, n)$ we see that each point of $\bar{D}_{h}$ may be uniquely represented by a pair of integers. We take as the boundary $\Gamma_{h}$ of $\bar{D}_{h}$ the points in the top two rows of $\bar{D}_{h}$; that is, $\Gamma_{h}$ consists of the points $(k, 0)$ for $k=1,2, \cdots, N-1$ and $(k, 1)$ for $k=0,1, \cdots, N-1$. The interior region $D_{h}$ is the set of points of $\bar{D}_{h}$ which do not belong to the boundary $\Gamma_{h}$.

At each point $(k, n)$ of $D_{h}$ we define a difference operator $L_{h}$, operating on any function $U$ defined on $\bar{D}_{h}$, by

$$
\begin{aligned}
L_{h} U_{k, n}= & \frac{1}{\lambda_{n-1} \lambda_{n}}\left(\frac{2 \lambda_{n}}{\lambda_{n-1}+\lambda_{n}} U_{k+1, n-2}+\frac{2 \lambda_{n-1}}{\lambda_{n-1}+\lambda_{n}} U_{k, n}-U_{k, n-1}-U_{k+1, n-1}\right) \\
& +a_{k, n} \frac{1}{h}\left(U_{k+1, n-1}-U_{k, n-1}\right)+b_{k, n} \frac{1}{\lambda_{n-1}+\lambda_{n}}\left(U_{k+1, n-2}-U_{k, n}\right) \\
& +c_{k, n} U_{k, n},
\end{aligned}
$$


where $\lambda_{n}=y_{n}-y_{n-1}$ and $U_{k, n}=U\left(x_{k}+n h / 2,-y_{n}\right), a_{k, n}=a\left(x_{k}+n h / 2,-y_{n}\right)$, etc. The difference problem corresponding to the Cauchy problem is that of finding a solution to

$$
L_{h} U=f
$$

on $D_{h}$, which takes on the initial values

$$
\begin{aligned}
U_{k, 0} & =\psi_{1}\left(x_{k}\right) ; & k & =1,2, \cdots, N-1, \\
U_{k, 1} & =\psi_{1}\left(x_{k}\right)-y_{1} \psi_{2}\left(x_{k}\right) ; & k & =0,1, \cdots, N-1 .
\end{aligned}
$$

Theorem 2.1. Let $u$ be of class $C^{2}(\bar{D})$. Then at each point of $D_{h}, L_{h} u \rightarrow L u$ uniformly as $h \rightarrow 0$.

Proof. By Taylor's theorem, we find that at a point $\left(x,-y_{n}\right)$ of $D_{h}$,

$$
\begin{aligned}
\left|L u-L_{h} u\right| \leqq & \left|K\left(-y_{n}\right)+\frac{h^{2}}{4 \lambda_{n-1} \lambda_{n}}\right|\left|\frac{\partial^{2} u}{\partial x^{2}}\right|+\frac{h^{2}}{4 \lambda_{n-1} \lambda_{n}} \epsilon_{1}+\frac{h}{2 \lambda_{n-1}} \epsilon_{2} \\
& +\left(1+\frac{\lambda_{n}}{\lambda_{n-1}}\right) \epsilon_{3}+|a| \epsilon_{4}+|b| \epsilon_{5} .
\end{aligned}
$$

Here the functions denote values at $\left(x,-y_{n}\right)$. The quantities $\epsilon_{1}, \epsilon_{2}, \epsilon_{3}, \epsilon_{4}$ and $\epsilon_{5}$ are the moduli of continuity of $\partial^{2} u / \partial x^{2}, \partial^{2} u / \partial x \partial y, \partial^{2} u / \partial y^{2}, \partial u / \partial x$ and $\partial u / \partial y$, respectively.

From estimates obtained in [1], we see that each term on the right side of (2.8) tends to zero uniformly as $h \rightarrow 0$.

3. A maximum principle. We denote difference quotients by subscripts, with unbarred subscripts indicating forward difference quotients and barred subscripts indicating backward difference quotients, as follows:

$$
\begin{aligned}
U_{x_{k, n}} & =\frac{1}{h}\left(U_{k+1, n}-U_{k, n}\right), & U_{\tilde{x}_{k, n}} & =\frac{1}{h}\left(U_{k, n}-U_{k-1, n}\right), \\
U_{y_{k, n}} & =\frac{1}{\lambda_{n-1}+\lambda_{n}}\left(U_{k+1, n-2}-U_{k, n}\right), & U_{\tilde{y}_{k, n}} & =\frac{1}{\lambda_{n+1}+\lambda_{n+2}}\left(U_{k, n}-U_{k-1, n+2}\right), \\
U_{s_{k, n}} & =\frac{1}{\lambda_{n}}\left(U_{k+1, n-1}-U_{k, n}\right), & U_{\bar{s}_{k, n}} & =\frac{1}{\lambda_{n+1}}\left(U_{k, n}-U_{k-1, n+1}\right), \\
U_{t_{k, n}} & =\frac{1}{\lambda_{n}}\left(U_{k, n-1}-U_{k, n}\right), & U_{i_{k, n}} & =\frac{1}{\lambda_{n+1}}\left(U_{k, n}-U_{k, n+1}\right) .
\end{aligned}
$$

In terms of difference quotients, (2.5) becomes

$$
\begin{aligned}
L_{h} U_{k, n}= & \frac{1}{\lambda_{n-1}+\lambda_{n}}\left(U_{s_{k, n-1}}-U_{s_{k, n}}+U_{t_{k+1, n-1}}-U_{t_{k, n}}\right) \\
& +a_{k, n} U_{x_{k, n-1}}+b_{k, n} U_{y_{k, n}}+c_{k, n} U_{k, n} .
\end{aligned}
$$


Let $(k, n)$ be any point of $D_{h}$, and consider the sum

$$
\sum_{T_{h}}\left(\lambda_{j-1}+\lambda_{j}\right) L_{h} U_{i, j}
$$

where $T_{h}$ is the set of points of $D_{h}$ which lie in the closed region bounded by the characteristics passing through $(k, n)$ and the line $y=-y_{2}$. Substituting the expression (3.1) into (3.2) we find that the contribution of the terms in $U$, and $U_{t}$ is given by

$$
\sum_{i=1}^{n-1}\left(U_{\bar{z}_{k+i, 0}}+U_{\bar{t}_{k+i}, 0}\right)-\sum_{j=2}^{n}\left(U_{s_{k+n-j, j}}+U_{t_{k, j}}\right) .
$$

The second of the above sums, in turn, may be written

$$
\begin{aligned}
\sum_{j=2}^{n}\left(U_{s_{k+n-j, j}}+U_{t_{k, j}}\right)=- & \sum_{j=1}^{n-1}\left(\frac{1}{\lambda_{j}}-\frac{1}{\lambda_{j+1}}\right)\left(U_{k+n-j, j}+U_{k, j}\right) \\
& +\frac{1}{\lambda_{1}}\left(U_{k+n-1,1}+U_{k, 1}\right)-\frac{2}{\lambda_{n}} U_{k, n} .
\end{aligned}
$$

In (3.2), the sum involving $U_{x}$ is first taken over the points in $T_{h}$ for which $y=-y_{m}$. For each $m$ with $m=2,3, \cdots, n$, these sums are of the form

$$
\begin{aligned}
\left(\lambda_{m-1}+\lambda_{m}\right) & \sum_{i=0}^{n-m} a_{k+i, m} U_{x_{k+i, m-1}}=-\left(\lambda_{m-1}+\lambda_{m}\right) \\
\cdot & \left\{\sum_{i=1}^{n-m} a_{\tilde{x}_{k+i, m}} U_{k+i, m-1}-\frac{1}{h} a_{k+n-m, m} U_{k+n-m+1, m-1}+\frac{1}{h} a_{k, m} U_{k, m-1}\right\} .
\end{aligned}
$$

We thus have

$$
\begin{aligned}
\sum_{T_{k}}\left(\lambda_{j-1}+\lambda_{j}\right) a_{i, j} U_{x_{i, j-1}} & =-\sum_{T_{h^{\prime}}}\left(\lambda_{j}+\lambda_{j+1}\right) a_{\bar{x}_{i, j+1}} U_{i, j} \\
& +\frac{1}{h} \sum_{j=1}^{n-1}\left(\lambda_{j}+\lambda_{j+1}\right)\left(a_{k+n-j-1, j+1} U_{k+n-j, j}-a_{k, j+1} U_{k, j}\right),
\end{aligned}
$$

where $T_{h}^{\prime}$ is the set of points of $\bar{D}_{h}$ which lie in the closed region bounded by the characteristics passing through $(k+1, n-2)$ and the line $y=-y_{1}$. Similarly, summing the terms involving $U_{y}$, we find that

$$
\begin{aligned}
\sum_{T_{h}}\left(\lambda_{j-1}+\lambda_{j}\right) b_{i, j} U_{y_{i}, j} & =-\sum_{T_{k^{\prime}}}\left(\lambda_{j+1}+\lambda_{j+2}\right) b_{\tilde{y}_{i, j}} U_{i, j} \\
& -\sum_{j=1}^{n-1}\left(b_{k+n-j, j} U_{k+n-j, j}+b_{k, j} U_{k, j}\right)+\sum_{i=1}^{n-1} b_{k+i-1,2} U_{k+i, 0} \\
& +\sum_{i=1}^{n-2} b_{k+i, 1} U_{k+i, 1}+b_{k+n-1,1} U_{k+n-1,1}+b_{k, 1} U_{k, 1}-b_{k, n} U_{k, n}
\end{aligned}
$$


Substituting these expressions into (3.2), we obtain

$$
\begin{aligned}
\sum_{T_{h}}\left(\lambda_{j-1}+\right. & \left.\lambda_{j}\right) L_{h} U_{i, j}=\sum_{T_{h^{\prime}}}\left\{\left(\lambda_{j-1}+\lambda_{j}\right) c_{i, j}-\left(\lambda_{j}+\lambda_{j+1}\right) a_{\bar{x}_{i, j+1}}\right. \\
& \left.\left.+\sum_{j+1}+\lambda_{j+2}\right) b_{\bar{y}_{i, j}}\right\} U_{,} \\
& \left.+\frac{1}{\lambda_{j}}-\frac{1}{\lambda_{j+1}}+\frac{\lambda_{j}+\lambda_{j+1}}{h} a_{k+n-j-1, j+1}-b_{k+n-j, j}\right\} U_{k+n-j, j} \\
& +\sum_{j=1}^{n-1}\left\{\frac{1}{\lambda_{j}}-\frac{1}{\lambda_{j+1}}-\frac{\lambda_{j}+\lambda_{j+1}}{h} a_{k, j+1}-b_{k, j}\right\} U_{k, j} \\
& +\sum_{j=2}^{n-1}\left(\lambda_{j-1}+\lambda_{j}\right)\left(c_{k+n-j, j} U_{k+n-j, j}+c_{k, j} U_{k, j}\right) \\
& +\sum_{i=1}^{n-1}\left(U_{\bar{s}_{k+i, 0}}+U_{\bar{t}_{k+i, 0}}+b_{k+i-1,2} U_{k+i, 0}\right) \\
& +\sum_{i=1}^{n-2}\left(b_{k+i, 1}-\lambda_{1} c_{k+i, 1}\right) U_{k+i, 1}-\left(\frac{1}{\lambda_{1}}-b_{k+n-1,1}\right) U_{k+n-1,1} \\
& -\left(\frac{1}{\lambda_{1}}-b_{k, 1}\right) U_{k, 1}+\left\{\frac{2}{\lambda_{n}}-b_{k, n}+\left(\lambda_{n-1}+\lambda_{n}\right) c_{k, n}\right\} U_{k, n} .
\end{aligned}
$$

In the first sum on the right side of (3.3), we take $\lambda_{0}=0$.

For convenience, let us define the sets $D_{h}^{\prime}$ and $D_{h}^{*}$ by

$$
D_{h}^{\prime}=\bar{D}_{h} \cap D, \quad D_{h}^{*}=\left\{\left(x,-y_{n}\right) \in \bar{D}_{h} \mid n=1,2, \cdots, N-1\right\} .
$$

TheOREM 3.1. Let $L_{h} U \leqq 0$ on $D_{h}$ with

$$
U_{k, 0} \leqq 0, \quad U_{\bar{s}_{k, 0}} \geqq 0, \quad U_{i_{k, 0}} \geqq 0 ; \quad k=1,2, \cdots, N-1 .
$$

Assume that the conditions

(3.5) $\quad c_{k, n} \leqq 0$ on $D_{h}^{*}$,

(3.6) $b_{k, 2} \leqq 0$,

$k=0,1, \cdots, N-1$.

(3.7) $\quad b_{k, 1}-\lambda_{1} c_{k, 1} \leqq 0$,

$k=1,2, \cdots, N-2$.

(3.8) $1 / \lambda_{1}-b_{k, 1} \geqq 0$,

$k=0,1, \cdots, N-1$,

(3.9) $2 / \lambda_{n}-b_{k, n}+\left(\lambda_{n-1}+\lambda_{n}\right) c_{k, n}>0$ on $D_{h}$,

(3.10) $1 / \lambda_{n}-1 / \lambda_{n+1}+\left(\lambda_{n}+\lambda_{n+1}\right) a_{k-1, n+1} / h-b_{k, n} \leqq 0$ on $D_{h}^{*}$,

(3.11) $1 / \lambda_{n}-1 / \lambda_{n+1}-\left(\lambda_{n}+\lambda_{n+1}\right) a_{k, n+1} / h-b_{k, n} \leqq 0$ on $D_{h}^{*}$,

(3.12) $\left(\lambda_{n-1}+\lambda_{n}\right) c_{k, n}-\left(\lambda_{n}+\lambda_{n+1}\right) a_{\tilde{x}_{k, n+1}}-\left(\lambda_{n+1}+\lambda_{n+2}\right) b_{\bar{y}_{k, n}} \leqq 0$ on $D_{h}^{\prime}$, are satisfied. Then the maximum of $U$ is attained on the boundary $\Gamma_{h}$.

Proof. Let us denote the maximum value of $U$ on $\Gamma_{h}$ by $M$. Then $M \leqq 0$ 
by (3.4). Suppose that the maximum is not attained on the boundary. Then it must be attained at some point $Q$ in the interior $D_{h}$. If $U(Q)>0$, then there is a point $P$ in $D_{h}$ such that $U(P)>0$ and $U \leqq 0$ at every point in $\bar{D}_{h}$ above $P$. Solving for $U_{k, n}=U(P)$ in (3.3) we find that by the hypotheses of the theorem, $U(P) \leqq 0$, which contradicts the assumption that $U(P)>0$. If $U(Q) \leqq 0$, then we consider the function $U_{1}=U-M$, which satisfies $L_{h} U_{1} \leqq 0$ and the conditions (3.4). Furthermore, $U_{1}(Q)>0$ by hypothesis. Hence there is a point $P$ in $D_{h}$ such that $U_{1}(P)>0$ and $U_{1} \leqq 0$ at every point in $\bar{D}_{h}$ above $P$. Again, the hypotheses of the theorem lead us to the contradictory result, $U_{1}(P) \leqq 0$. Therefore the maximum of $U$ must be attained on the boundary.

We next find, in terms of the coefficients of the differential equation (1.1), sufficient conditions for (3.5) through (3.12) to be satisfied. First of all, it is clear that if

$$
\begin{aligned}
& c \leqq 0 \text { on } D, \\
& b<0 \text { if }-\delta \leqq y \leqq 0 \text { on } \bar{D} \text { for some } \delta>0,
\end{aligned}
$$

then the conditions (3.5) through (3.9) are satisfied for all $h$ sufficiently small.

THEOREM 3.2. Suppose that

$$
\frac{d}{d y}\left[(-K)^{1 / 2}\right] \pm a-b(-K)^{1 / 2}<0
$$

for $y<0$ on $\bar{D}$, and

$$
y a(x, y)[-K(y)]^{1 / 2} \rightarrow 0 \text { as } y \rightarrow 0
$$

uniformly on $\bar{D}$. Let the function $H^{\prime}$, defined by (2.3), have the form

$$
H^{\prime}(x)=x^{-\alpha} \tilde{H}(x)
$$

near $x=0$, where $0<\alpha<1$ and $\tilde{H}$ is a function having three continuous derivatives with $\tilde{H}(x) \geqq m>0$ for $x \geqq 0$. Then the conditions (3.10) and (3.11) are satisfied for $h$ sufficiently small.

We omit the proof, which is the same as the proof of Theorem 3.5 of [1].

TheOREM 3.3. Let

$$
c-\frac{\partial a}{\partial x}-\frac{\partial b}{\partial y}<0
$$

on $D$. Then for each $\delta>0$, the condition (3.12) is satisfied for h sufficiently small, provided $y_{n} \geqq \delta$.

Proof. Since $a$ and $b$ are assumed to be continuously differentiable on $\bar{D}$, the difference quotients $a_{\varepsilon}$ and $b_{\bar{y}}$ tend uniformly to $\partial a / \partial x$ and $\partial b / \partial y$ as $h \rightarrow 0$. But for $y_{n} \geqq \delta$, 


$$
\frac{\lambda_{n}+\lambda_{n+1}}{\lambda_{n-1}+\lambda_{n}} \rightarrow 1 \text { and } \frac{\lambda_{n+1}+\lambda_{n+2}}{\lambda_{n-1}+\lambda_{n}} \rightarrow 1
$$

as $h \rightarrow 0$. This implies that the left side of (3.12) divided by $\lambda_{n-1}+\lambda_{n}$ can be made uniformly close to the left side of (3.18) for $h$ small, provided $y_{n} \geqq \delta$.

We note that the conditions (3.13), (3.14), (3.15) and (3.18) are essentially the conditions, obtained by Weinberger [2] and Protter [3], under which the differential equation has the maximum property. It is also of interest to observe that the restriction (3.16) is precisely the condition which Protter [4] found guarantees the solvability of the Cauchy problem for the differential equation (1.1).

4. The existence of the solution to the difference equation.

THEOREM 4.1. Let the conditions (3.5) through (3.12) be satisfied. Then the difference equation (2.6) with initial conditions (2.7) has a unique solution for arbitrary values of $f, \psi_{1}$ and $\psi_{2}$.

Proof. The system (2.6) and (2.7) is a system of $P$ linear algebraic equations in the $P$ unknown values of $U$ on $D_{h}$. We first consider a solution $V$ of the homogeneous system which results when we set $f \equiv \psi_{1} \equiv \psi_{2} \equiv 0$. For this system, the hypotheses of Theorem 3.1 are satisfied by both the functions $V$ and $-V$. Therefore the maximum principle implies that $V \equiv 0$ on $D_{h}$. But this means that for arbitrary values of $f, \psi_{1}$ and $\psi_{2}$, the system has a unique solution.

\section{A priori bounds.}

THEOREM 5.1. Let the conditions (3.5) through (3.12) be satisfied for $h$ sufficiently small. Let $U$ be any function defined on $\bar{D}_{h}$ and let

$$
\begin{aligned}
& N=\max \left\{\max _{1 \leqq k \leqq N-1}\left(\left|U_{k, 0}\right|,\left|U_{\bar{t}_{k, 0}}\right|,\left|U_{\bar{t}_{k, 0}}\right|\right), \max _{D_{h}}\left|L_{h} U\right|\right\}, \\
& Y=\max _{\bar{D}}|y| .
\end{aligned}
$$

Then for $h$ sufficiently small,

$$
|U| \leqq N e^{\mu Y}
$$

on $D_{h}$, for some fixed $\mu>0$.

Proof. Choose $\mu$ so large that $\mu \geqq 1$, and on $\bar{D}$

$$
\mu^{2}-2|b| \mu-4|c| \geqq 1 .
$$

We now define a mesh function $E(y)$ on $\bar{D}_{h}$ by

$$
E(0)=1, \quad E\left(-y_{n}\right)=\left(1+\mu \lambda_{n}\right) E\left(-y_{n-1}\right) .
$$

Then $E(y) \geqq 1$ and 


$$
E\left(-y_{n}\right)=\prod_{j=1}^{n}\left(1+\mu \lambda_{j}\right) \leqq \exp \left(\sum_{j=1}^{n} \mu \lambda_{j}\right) \leqq e^{\mu Y} .
$$

It is easily seen that $E_{\bar{s}_{k, 0}}=E_{\bar{t}_{k, 0}}=-\mu \leqq-1$. Finally,

$$
\begin{aligned}
L_{h} E\left(-y_{n}\right)=\left\{\frac{2 \lambda_{n-1}}{\lambda_{n-1}+\lambda_{n}} \mu^{2}-(1\right. & \left.+\frac{\lambda_{n-1} \lambda_{n}}{\lambda_{n-1}+\lambda_{n}} \mu\right) b_{k, n} \mu \\
& \left.+\left(1+\mu \lambda_{n}\right)\left(1+\mu \lambda_{n-1}\right) c_{k, n}\right\} E\left(-y_{n-2}\right) .
\end{aligned}
$$

If we now choose $h$ so small that $\mu \lambda_{n} \leqq 1$, we have

$$
L_{h} E\left(-y_{n}\right)>\mu^{2}-2|b| \mu-4|c| \geqq 1 \text {. }
$$

We now consider the functions

$$
\begin{aligned}
V & =U-N E, \\
W & =-U-N E .
\end{aligned}
$$

From the definition of $N$ and $E$ it is easily seen that both $V$ and $W$ satisfy the hypotheses of Theorem 3.1. Therefore, the maxima of $V$ and $W$ are attained on the boundary. Since $V \leqq 0$ and $W \leqq 0$ on the boundary, the bound (5.1) holds for $U$.

\section{The convergence theorem.}

THEOREM 6.1. Suppose that the differential equation (1.1) has a solution $u$ satisfying the initial conditions (1.3), such that $u$ is of class $C^{2}(\bar{D})$. Assume that the conditions (3.5) through (3.12) are satisfied for $h$ sufficiently small. Then the solution of the difference equation (2.6) with initial conditions (2.7) tends uniformly to $u$ as $h \rightarrow 0$.

Proof. Given $\epsilon>0$, by Theorem 2.1 we may choose $h_{0}>0$ so small that for $0<h \leqq h_{0}$,

$$
\left|L u-L_{h} u\right|<\epsilon e^{-\mu Y},
$$

where $\mu$ and $Y$ are as defined in Theorem 5.1. But since $L u=L_{h} U=f$ on $D_{h}$, this estimate may be written

$$
\left|L_{h}(U-u)\right|<\epsilon e^{-\mu Y} \text {. }
$$

Furthermore,

$$
\begin{aligned}
U_{k, 0} & =u_{k, 0}=\psi_{k}\left(x_{k}\right), \\
U_{\bar{s}_{k, 0}} & =\frac{h}{2 y_{1}} \psi_{1}^{\prime}\left(x_{k}-\frac{h}{2} \theta_{1}\right)+\psi_{2}\left(x_{k}-\frac{h}{2}\right), \\
U_{\bar{t}_{k, 0}} & =\frac{h}{2 y_{1}} \psi_{1}^{\prime}\left(x_{k}+\frac{h}{2} \theta_{2}\right)+\psi_{2}\left(x_{k}+\frac{h}{2}\right),
\end{aligned}
$$


with $0<\theta_{1}<1,0<\theta_{2}<1$. On the other hand,

$$
\begin{aligned}
& u_{\bar{s}_{k, 0}}=\frac{h}{2 y_{1}} \frac{\partial u}{\partial x}\left(x_{k}-\frac{h}{2} \alpha_{1}, 0\right)+\frac{\partial u}{\partial y}\left(x_{k}-\frac{h}{2},-\beta_{1} y_{1}\right), \\
& u_{\bar{t}_{k, 0}}=-\frac{h}{2 y_{1}} \frac{\partial u}{\partial x}\left(x_{k}+\frac{h}{2} \alpha_{2}, 0\right)+\frac{\partial u}{\partial y}\left(x_{k}+\frac{h}{2}, \beta_{2} y_{1}\right),
\end{aligned}
$$

for some numbers $\alpha_{1}, \alpha_{2}, \beta_{1}, \beta_{2}$ between 0 and 1 . Therefore we see that

$$
\begin{aligned}
& \left|U_{\bar{s}_{k, 0}}-u_{\bar{s}_{k, 0}}\right|<\epsilon e^{-\lambda Y}, \\
& \left|U_{\bar{t}_{k, 0}}-u_{\bar{t}_{k, 0}}\right|<\epsilon e^{-\lambda Y},
\end{aligned}
$$

for $0<h \leqq h_{1}$. Applying Theorem 5.1 to the function $U-u$, we find that $|U-u|<\epsilon$ on $D_{h}$, if $h \leqq \min \left(h_{0}, h_{1}\right)$.

\section{BIBLIOGRAPHY}

1. H. Ogawa, On difference methods for the solution of a Tricomi problem, Trans. Amer. Math. Soc. vol. 100 (1961) pp. 404-424.

2. H. F. Weinberger, A maximum property of Cauchy's problem, Ann. of Math. vol. 64 (1956) pp. 505-513.

3. M. H. Protter, A maximum principle for hyperbolic equations in a neighborhood of an initial line, Trans. Amer. Math. Soc. vol. 87 (1958) pp. 119-129.

4. - The Cauchy problem for a hyperbolic second order equation with data on the parabolic line, Canad. J. Math. vol. 6 (1954) pp. 435-446.

UNIVERSITY OF CALIFORNIA,

Riverside, California 